\title{
Timing of Neutrophil Depletion Influences Long-Term Neuroprotection in Neonatal Rat Hypoxic-Ischemic Brain Injury
}

\author{
CHARLES PALMER, REBECCA L. ROBERTS, AND PAMELA I. YOUNG \\ Department of Pediatrics, The Milton S. Hershey Medical Center, Penn State University College of \\ Medicine, Hershey, Pennsylvania, 17033 U.S.A.
}

\begin{abstract}
In neonatal rats, neutrophils do not accumulate in ischemic brain parenchyma to the extent that they do in adult rodents. They are also confined to the intravascular compartment during the first few hours of recovery. However, neonatal rats rendered neutropenic have less brain swelling after a hypoxic-ischemic (HI) insult. In this study, we used the Rice-Vannucci model of HI brain injury in 7-d-old rats, and we depleted neutrophils before injury in one group and 4-8 h after injury in another group to determine 1) whether neutrophils contribute to cerebral atrophy, 2) whether neutropenia induced within $8 \mathrm{~h}$ after recovery from $\mathrm{HI}$ is neuroprotective, and 3) whether neutropenia preserved energy metabolites during the HI insult. Brain energy metabolites were measured at $0 \mathrm{~h}$ and $6 \mathrm{~h}$ of recovery. Brain atrophy was measured morphometrically on brain slices at $2 \mathrm{wk}$ of recovery. In 67 rats, we found that neutropenia induced before the HI insult, but not after $\mathrm{HI}$, reduced brain swelling at $42 \mathrm{~h}$ of recovery by
\end{abstract}

Neutrophils contribute to ischemic brain injury through their ability to reduce microvascular flow $(1,2)$ and release cytotoxic agents into the vasculature and brain parenchyma $(3,4)$. Early accumulation of neutrophils in blood vessels has been reported within hours after cerebral ischemia in adult animals $(5,6)$. Parenchymal accumulation in adult animals occurs between 6 and $48 \mathrm{~h}$ post insult, during the period while brain injury is evolving (6-9). Interestingly, neuroprotection can be achieved with antineutrophil strategies initiated after the HI insult in adult animals (10-12).

Neonates have a diminished ability to mount a neutrophil response compared with adults; and neonatal neutrophils have a diminished tendency to extravasate from blood vessels (13, 14). Studies on neonatal neutrophils have documented diminished chemotaxis $(15,16)$ diminished adherence (17), and deficiencies in the expression of adhesion molecules and complement receptors $(14,18)$. However, neutrophils are implicated in ischemic brain injury in the neonatal rat because neutropenia induced before HI reduces brain swelling (19).

Received August 11, 2003; accepted November 27, 2003.

Correspondence: Charles Palmer, M.B., Ch.B., Department of Pediatrics, P.O. Box 850, MC H085, The Milton S. Hershey Medical Center, 500 University Dr., Hershey, PA 17033, U.S.A.; e-mail: cpalmer@psu.edu

Supported by Grant 30704-09 from the National Institutes of Health. about $75 \%(p<0.001)$. In another 60 rats, we found that cerebral atrophy was reduced by $61 \%$ provided that neutropenia was induced before HI $(p<0.05)$. Total adenine nucleotides were better preserved in the neutropenic rats at the end of the $\mathrm{HI}$ insult ( $0 \mathrm{~h}$ recovery); $p<$ 0.05 . We conclude that neutrophils do contribute to vascular dysfunction either during the HI insult or early hours $(<4-8 \mathrm{~h})$ of recovery. Antineutrophil strategies initiated after this time are unlikely to be protective in the neonatal rat. (Pediatr Res 55: 549-556, 2004)

\section{Abbreviations \\ HI, hypoxic-ischemic, hypoxia-ischemia ANS, antineutrophil serum PVL, periventricular leukomalacia TAN, total adenine nucleotides}

In the neonatal rat, neutrophils are seen in blood vessels during the first few hours after HI $(19,20)$. There are very few studies that count neutrophils in the neonatal parenchyma after ischemia and the resulting reports are variable. We were unable to find an accumulation of neutrophils in the injured parenchyma during the first $42 \mathrm{~h}$ of recovery (19). However, other investigators using different detection methods found neutrophils were accumulating in the injured parenchyma $12-72 \mathrm{~h}$ after the HI insult $(20,21)$. Bona et al. (20) reported a transient elevation at $12 \mathrm{~h}$ post $\mathrm{HI}$ and Benjelloun et al. (21) reported neutrophil infiltration starting at $24 \mathrm{~h}$ and peaking at 72-96 h. It is unknown whether these accumulating neutrophils in the parenchyma contribute to brain injury.

The predominant intravascular location of neutrophils in the early stages of reperfusion provokes the hypothesis that the intravascular neutrophils might contribute more to vascular injury than parenchymal injury. Accordingly, the reduction in brain swelling achieved with neutropenia might have addressed a predominantly vascular process. We have shown previously that a reduction of early brain swelling with a therapeutic intervention that did not cross the blood-brain barrier does not necessarily produce a reduction in cerebral infarction in this model (22). Therefore, the first aim of this study was to determine whether neutropenia would reduce cerebral atrophy. 
As the extent and implication of neutrophil extravasation remains unclear, we needed to determine whether antineutrophil strategies could be initiated during recovery from transient cerebral ischemia so that damage caused by infiltrating neutrophils could be evaluated. Thus, the second aim was to determine whether neutropenia could be delayed $6-8 \mathrm{~h}$ into recovery and still remain protective.

The intravascular location of neutrophils during the insult and early reperfusion period also suggests that neutrophils might impair red cell movement and oxygen delivery $(19,23)$. In the neonatal rat model of ischemic brain injury, transient ischemia is a key component of the insult, as hypoxia does not produce damage unless it is accompanied by ischemia (24). The combined insult (HI) produces a damaging depletion of energy metabolites in the neonatal brain that causes cellular necrosis and an ongoing process of injury that continues to evolve for days $(25,26)$. We hypothesized that the presence of neutrophils during hypoperfusion might contribute to the depletion in brain energy metabolism. Accordingly, the third objective was to evaluate the effect of neutrophils on brain high-energy phosphates at the end of a $\mathrm{HI}$ insult in the neonatal rat.

To achieve these aims, we studied three groups of 7-d-old rats that were subjected to a $\mathrm{HI}$ cerebral insult. One group was rendered neutropenic before the $\mathrm{HI}$ insult, another group was rendered neutropenic $6-8 \mathrm{~h}$ after $\mathrm{HI}$, and we then compared their outcomes against a control group with normal neutrophil counts.

We found that neutropenia induced before the $\mathrm{HI}$ insult did reduce long-term brain injury, however, when neutropenia was induced after the insult it was not protective. This finding suggests that intravascular neutrophils produce their damaging effects by impairing oxygen delivery. This is indicated by better preservation of high-energy phosphates at the end of the HI insult when neutrophils are depleted.

\section{METHODS}

Determining the time course of neutrophil depletion in the 7-d-old rat. Seven-day-old rat pups were weighed and injected subcutaneously with a $50 \%$ dilution of anti-neutrophil serum [anti-rat polymorphonuclear (PMN) antibody, Accurate Antibodies, Westbury, NY, U.S.A.] in a dose of $6 \mu \mathrm{L} / \mathrm{g}$ body weight. This antibody agglutinates rat PMN but not macrophages or thymocytes. Blood collected from the severed neck vessels was drained into blood collection micro-tubes containing EDTA. Blood smears were prepared from the sample and automated total nucleated cell counts and platelet counts were performed (Celldyne 400, Abbott Diagnostics, Abbott Park, IL, U.S.A.). A differential count was performed on the blood smear and the total white cell count was determined by correcting the automated count for nucleated red blood cells. The absolute neutrophil count per square millimeters of blood was obtained from groups of rats $(n=3-4)$ killed at 2, 4, 6, 8, and $24 \mathrm{~h}$ after administration of the antineutrophil serum and compared against normal age matched controls.

HI immature rat model (Rice-Vannucci model). For the HI immature rat model - the Rice-Vannucci model (27)—dated, pregnant, verified antigen-free Wistar rats were purchased from Charles River (Wilmington, MA, U.S.A.). HI injury to the right cerebral hemisphere of 7-d-old rats of either sex was produced by right common carotid artery ligation and exposure to hypoxia at $8 \%$ oxygen as previously described (19). All animal procedures were performed with approval of our institutional animal care committee.

Temperature effects of neutropenia. We measured the effect of neutropenia on core body temperature. Twelve 7-d-old rat pups were subjected to right common carotid artery ligation and $3 \mathrm{~h}$ of recovery with their dams. The antineutrophil serum was diluted 50\% in normal saline and injected in a dose of 6 $\mu \mathrm{L}$ per gram body weight. The antineutrophil serum injections were administered at the time of carotid ligation. The antibody induced neutropenia within $4-6 \mathrm{~h}$ in the immature rat. The pups were removed from their dams at $3.25 \mathrm{~h}$ of recovery from ligation and placed into syringe barrels housed within an incubator at $33.5^{\circ} \mathrm{C}$. The pups were fitted with rectal temperature probes (511, YSI Inc., Yellow Springs, OH, U.S.A.). They were monitored for $45 \mathrm{~min}$ to permit equilibration of body temperature to the incubator environment, $2.25 \mathrm{~h}$ of hypoxia, during which time $8 \%$ oxygen, balance nitrogen, was introduced into the syringe for the rats to breathe, and then $1 \mathrm{~h}$ of recovery in room air in the incubator. Temperature was recorded every $5 \mathrm{~min}$. We averaged the temperature recordings for the last 30-60 $\mathrm{min}$ of the measurement periods. Blood samples were taken at $42 \mathrm{~h}$ of recovery to confirm neutropenia.

Timing of neutropenia on brain swelling. Sixty-seven 7-dold rat pups were weighed, then the right common carotid artery was permanently ligated under halothane anesthesia. The pups were then randomized into three treatment groups so that rats allocated to the before-HI group received antineutrophil serum (ANS) immediately after carotid artery ligation. The anti-neutrophil serum (rabbit, anti-rat, PMN antibody from Accurate Antibodies) was diluted 50\% with normal saline and administered at $6 \mu \mathrm{L}$ per gram body weight subcutaneously. Four hours after receiving ANS, the rats were subjected to $2.25 \mathrm{~h}$ hypoxia in $8 \%$ oxygen (before-HI group). Then they were returned to their dams in room air. The after-HI group received the same dose of ANS immediately after hypoxia. The control group received normal rabbit serum (Sigma Chemical, St. Louis, MO, U.S.A.). Half of the control rats were treated before $\mathrm{HI}$ and the other immediately after HI, similar to the groups that received ANS. At the end of $2.25 \mathrm{~h}$ hypoxia, all surviving pups were returned to their dams and allowed to recover for $42 \mathrm{~h}$. At $42 \mathrm{~h}$ of recovery, the pups were killed by decapitation (before-HI, $n=18$; after-HI, $n=24$; control group, $n=25$ ). Blood was collected into heparinized Eppendorf tubes from severed neck vessels to confirm neutropenia. The brains were quickly removed and the posterolateral half of each cerebral hemisphere was removed and weighed in a tarred glass vial on a microanalytical balance. This region was chosen because it is frequently damaged in this model. After drying at $80^{\circ} \mathrm{C}$ for $72 \mathrm{~h}$, the vials were re-weighed. The difference in weight represented the water content and was expressed as a percentage $(\% \mathrm{WC})(28)$. The percentage dry weight $(\% \mathrm{DW})$ of each sample was determined according to the formula $(\% \mathrm{DW}$ $=100-\% \mathrm{WC})$. Swelling follows the influx of water into the 
right hemisphere. This is reflected in an increase in \%WC and a decrease in $\% \mathrm{DW}$. Right hemisphere swelling was calculated from the relative change in $\% \mathrm{DW}$ of the swollen right (R) compared with the normal left (L) hemisphere. Swelling was expressed as a percentage by the formula (\% DWL - \%DWR/ $\% \mathrm{DWL}) \times 100(19)$. A differential count was performed on blood smears from each rat and the percentage neutrophils calculated. In separate littermates, we confirmed that neutrophils were depleted $6 \mathrm{~h}$ after administration of the antineutrophil serum used in this experiment.

Timing of neutropenia on brain atrophy. Rat pups $(n=60)$ were divided into the same three experimental groups, treated with ANS, and subjected to the $\mathrm{HI}$ insult as described above for the determination of brain swelling. However, the rats were returned to their dams and allowed to recover for another $14 \mathrm{~d}$ before they were killed with a lethal dose of pentobarbital. Treatment groups were identified by ear clipping at $7 \mathrm{~d}$ of age, which incurred minimal bleeding. The brains were carefully removed and immersion fixed in FAM (1:8:1, formaldehyde, methanol, $1 \%$ acidic acid). After $14 \mathrm{~d}$ fixation, the brains were cut into five 2-mm-thick slices that were imaged with a computer imaging system using NIH Image 1.43 software. We calculated the area of each hemisphere from the digital images. Slices 1 and 5 were partial slices from the front and back of the brain, respectively, and not used for atrophy calculations. Slices 2, 3, and 4 were compared for treatment groups. Slices 3 and 4 represent the region of maximal injury in this model, and they were cut through the mamillary body on the base of the brain. As the left hemisphere (contralateral to ligation) remains morphometrically normal in size in this model (29), we calculated the percentage right hemisphere atrophy from the percentage reduction in the right hemisphere area compared with the left hemisphere area, according to the following formula: left - right/left $\times 100$, where left and right refer to left and right hemisphere areas, respectively. We compared the percentage in atrophy for slice 2, 3, and 4 individually and then determined the average difference by combining all the slices.

Measurement of high-energy metabolites. Rat pups were subjected to the standard $\mathrm{HI}$ insult as described above. Half the pups received ANS $4 \mathrm{~h}$ before hypoxia $(6 \mu \mathrm{L} / \mathrm{g}$ body weight, Accurate Antibodies). The other half received normal rabbit serum (Sigma Chemical) We confirmed in one or two rat pups from each litter that the antineutrophil serum did induce neutropenia. These control pups were killed at $24 \mathrm{~h}$ post treatment and their differential blood smears were checked for neutrophils to insure that they were neutropenic. After the $4 \mathrm{~h}$ recovery period following antineutrophil serum administration and carotid artery ligation, the pups were subjected to hypoxia at $8 \%$ oxygen in a $36.8^{\circ} \mathrm{C}$ water bath for $2.25 \mathrm{~h}$. To optimally preserve labile metabolites in the brain, rat pups were quick frozen in liquid nitrogen at either $0 \mathrm{~h}$ recovery or at $6 \mathrm{~h}$ of recovery. The frozen pups were stored at $-80^{\circ} \mathrm{C}$ until such time that each brain could be dissected from its skull in a cold box set at $-20^{\circ}$ and a sample of tissue (approximately $100 \mathrm{mg}$ ) removed from each cerebral hemisphere in the distribution area of the middle cerebral artery. The brain specimens were powdered under liquid nitrogen and the powders were weighed on a microanalytical balance and extracted into $3.0 \mathrm{M}$ perchloric acid as previously described $(25,30)$. The extracts were neutralized to a $\mathrm{pH}$ of 6.8 with $2.0 \mathrm{M}$ potassium carbonate, and maintained at $-80^{\circ} \mathrm{C}$ until the time of analysis. Concentrations of high-energy phosphate compounds [phospho-creatinine (P$\mathrm{Cr}$ ), ATP, ADP, and AMP] were determined using specific enzymatic fluorometric procedures $(30,31)$. We compared the neutropenic versus non-neutropenic rats at $0 \mathrm{~h}$ recovery $(n=$ 16 /group) and at $6 \mathrm{~h}$ of recovery ( $n=10-12$ /group). We also determined metabolite levels on 10 normal age-matched controls not subjected to hypoxia or ischemia.

\section{RESULTS}

Antineutrophil depletion time course. Figure 1 shows that administration of the antineutrophil serum reduced the absolute neutrophil count after $8 \mathrm{~h}$ from a normal level of $6180 \pm 1457$ per $\mathrm{mm}^{3}$ to $389 \pm 778$ (mean \pm SE). This is a fall of $94 \%$ by $8 \mathrm{~h}$ postinjection. At $4 \mathrm{~h}$ postinjection, the neutrophil count had already fallen by $75 \%$. The rats were still neutropenic $24 \mathrm{~h}$ after receiving the antineutrophil serum.

Neutropenia before HI reduces brain swelling. Neutropenia induced before HI (before-HI group) reduced brain swelling by $77 \%$ (Fig. 2). The right hemisphere swelled $13.7 \pm$ $9.1 \%$ (mean $\pm \mathrm{SD}$ ) in the control group and $10.2 \pm 9.5 \%$ in the after-HI group. In the before-HI group, the right hemisphere swelled only $3.2 \pm 4.2 \%(p<0.001$, ANOVA, versus other groups). Thus, neutropenia induced before cerebral HI (before$\mathrm{HI})$ reduced brain swelling at $42 \mathrm{~h}$ recovery by $75 \%(p=$ 0.0001 ). In contrast, neutropenia induced within $4-8 \mathrm{~h}$ after HI (after-HI) was not protective.

Differential counts of peripheral blood smears taken at the time of sacrifice ( $42 \mathrm{~h}$ recovery) showed that those rats that received ANS were still neutropenic. The percentage neutrophils in the control group was $26.9 \pm 12.8 \%$, the before-HI group was $0.7 \pm 2.4 \%$, and the after-HI group $0.7 \pm 1.4 \%$

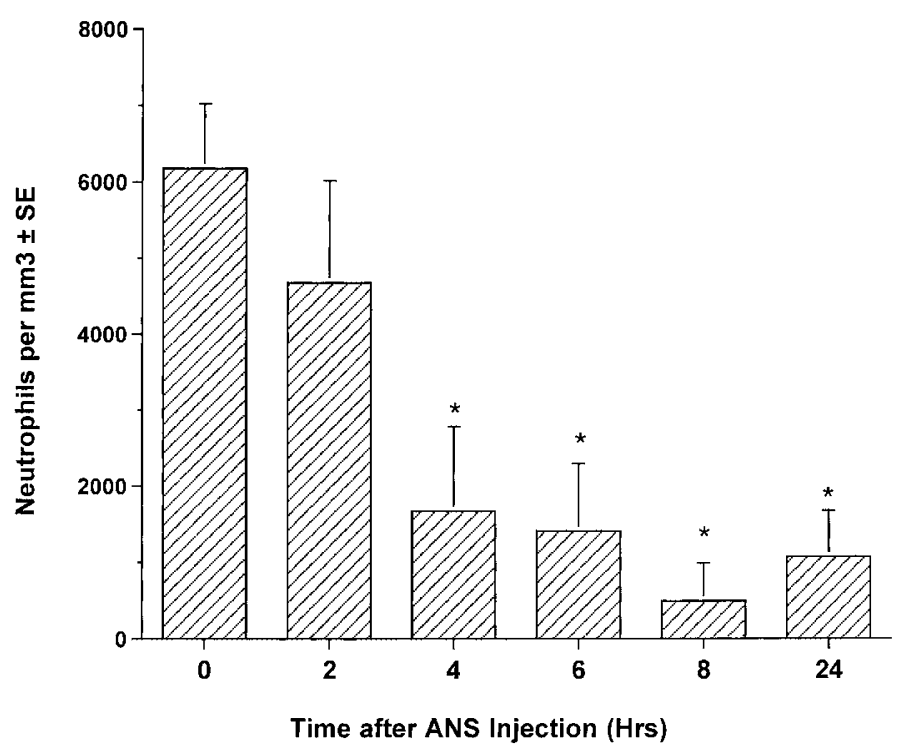

Figure 1. Time course of rat peripheral blood neutrophil depletion after injection with ANS at $0 \mathrm{~h}$. Eight hours after administration of ANS, neutrophil counts had fallen $94 \%$. *Neutrophil counts significantly lower than at $0 \mathrm{~h}(p<$ $0.05, t$ test). 


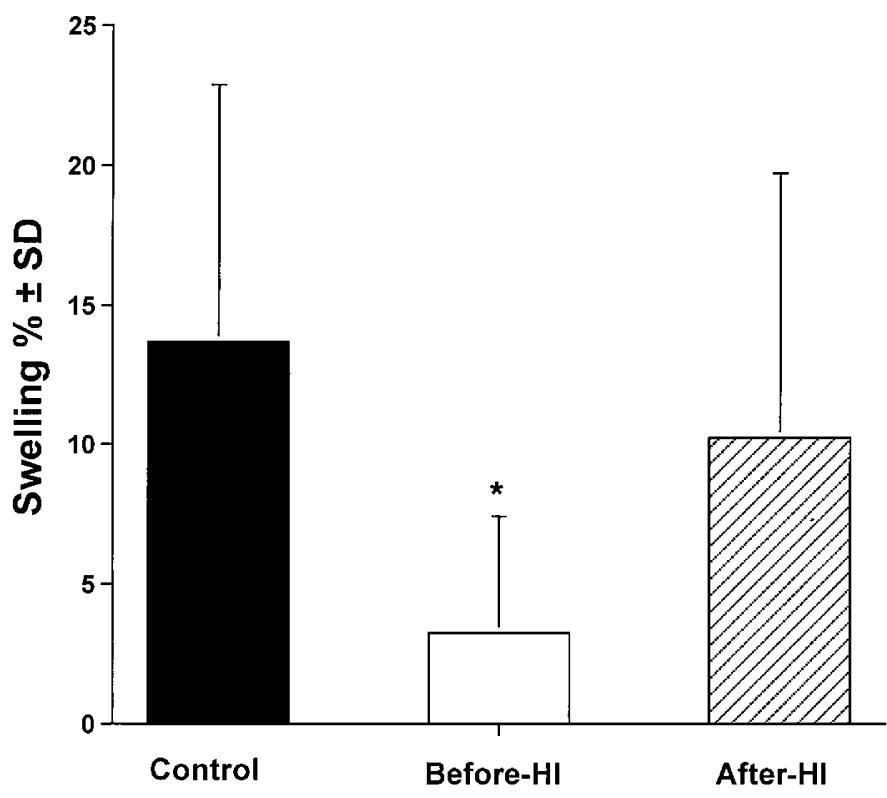

Figure 2. Bar chart showing that brain swelling was reduced in the before-HI group only. (Control group, $n=25$; before-HI group, $n=15$; after-HI group, $n=24)$.

( $p<0.001$, control versus before-HI and after-HI group, ANOVA).

Neutropenia before $H I$ reduces brain atrophy. The before-HI group had less atrophy in all three slices (significant for the posterior slices 3 and 4 only) (Fig. 3). The total atrophy calculated from averaging the results taken from slices 2, 3, and 4 showed that the control group (non-neutropenic rats, $n=$ 21) had $18.19 \pm 15.5 \%$ atrophy, and the after-HI group $(n=$ 22 ) had $21.7 \pm 17.8 \%$ atrophy. In contrast, the before-HI group had $7.15 \pm 11.4 \%$ atrophy. This indicates that neutropenia

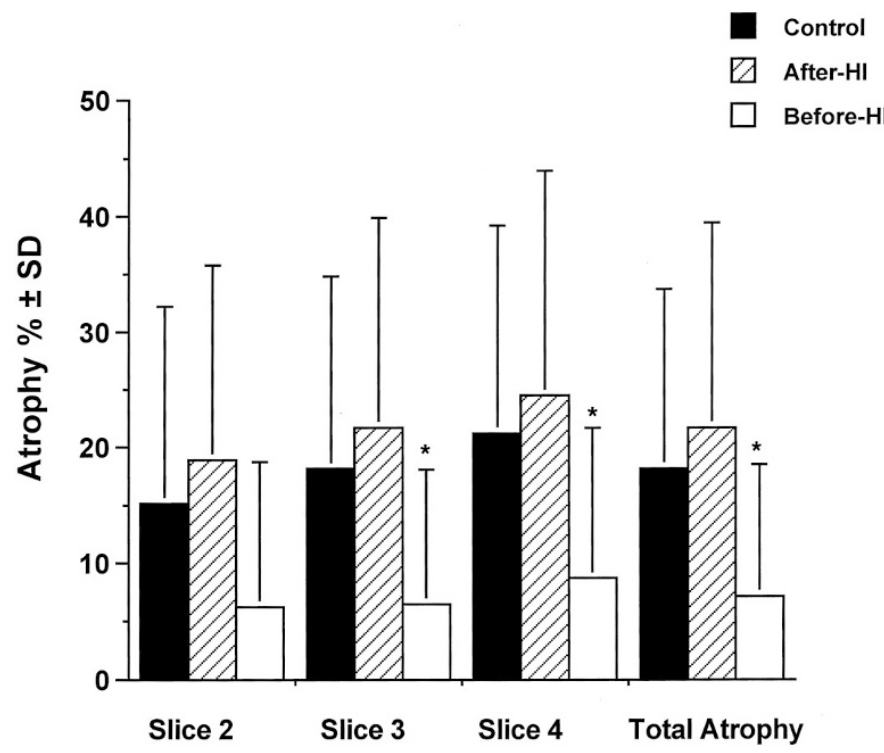

Figure 3. Bar graphs showing right hemisphere atrophy for each 2-mm-thick brain slice. Slices 3 and 4 were taken at the level (of the mamillary body) where maximum injury is usually seen in this model. Slice 2 is anterior. Total atrophy was reduced in the before-HI group only $[p=0.03$ s control and $<0.01$ vs before-HI group (control group, $n=20$; after-HI group, $n=22$; before-HI group, $n=17)$ ]. induced before cerebral HI reduced right hemisphere atrophy by $\pm 61 \%$ ( $p=0.03$, ANOVA, pre versus control), whereas neutropenia induced within $4-8 \mathrm{~h}$ after HI (after-HI group) was not protective. When the before-HI group was compared with the after-HI group in terms of total atrophy, the difference was also significant $(p \leq 0.01)$.

ATP and energy metabolites. We compared the energy metabolite levels of the neutropenic and non-neutropenic rats at 0 and 6 h of recovery (Table 1). The levels of brain ATP in normal 7-d-old rats was $2.29 \pm 0.18 \mathrm{mmol} / \mathrm{kg}$ (mean $\pm \mathrm{SD}$ ). In the non-neutropenic rats at $0 \mathrm{~h}$ recovery, ATP levels were $0.88 \pm 0.59$ in the ipsilateral (damaged) hemisphere, whereas in the neutropenic rats ATP levels trended lower at $1.3 \pm 0.76$ ( $p=0.06$, Mann-Whitney $U$ test). Seven of the 16 neutropenic rats had ATP levels at $0 \mathrm{~h}$ recovery that were normal (within 2 $\mathrm{SD})$, compared with only 2 of the 14 non-neutropenic rats ( $p=$ $\left.0.04, \chi^{2}\right)$. At 6 h of recovery, ATP levels had returned to within $2 \mathrm{SD}$ of the mean $(1.93-2.65 \mathrm{mmol} / \mathrm{kg})$ in all 12 neutropenic rats and 7 of the 10 non-neutropenic rats. There was no significant difference between the neutropenic and nonneutropenic rats at $6 \mathrm{~h}$ of recovery for any metabolite (Fig. 4).

The normal range for TAN was $2.7 \pm 0.22 \mathrm{mmol} / \mathrm{kg}$. At $0 \mathrm{~h}$ recovery in the ipsilateral hemisphere of neutropenic rats, TAN levels were $2.02 \pm 0.56 \mathrm{mmol} / \mathrm{kg}$, and only $1.55 \pm 0.56$ in the non-neutropenic rats ( $p=0.02$, Mann-Whitney $U$ test) (Fig. 5). There was no difference in the other metabolites measured at $0 \mathrm{~h}$ recovery (Table 1$)$. These data suggest that neutropenia induced before the HI insult helps to preserve TAN during the insult and by $6 \mathrm{~h}$ of recovery cerebral energy metabolites returned to normal in the rats from both groups.

Effects of neutropenia on body temperature. The ANS had no significant effect on body temperature. Table 2 illustrates the results for the temperature measurements.

\section{DISCUSSION}

We initially established that neutrophils in the peripheral blood of the 7-d-old rat can be depleted by 75-93\% at 4-8 h after administration of antineutrophil serum (Fig. 1). This finding enabled us to evaluate the differences in neuroprotection that might be achieved if neutropenia was induced before the cerebral insult or, alternately, within $4-8 \mathrm{~h}$ after the insult, by administration of antineutrophil serum at the beginning of recovery from cerebral $\mathrm{HI}$.

We have confirmed our previous findings, in that neutropenia induced before HI brain injury reduces brain swelling in the 7-d-old rat (19). In contrast, neutropenia induced after the insult did not reduce brain swelling (Fig. 2). We have also shown that cerebral atrophy is reduced if neutropenia is induced before the insult, but not if neutropenia is induced 4-8 $\mathrm{h}$ after the insult (Fig. 3). This establishes the important role that neutrophils play in the pathogenesis of long-term HI brain injury in the neonatal rat. It also establishes that neutrophils produce their damaging effects during either the ischemic insult or during the first $4-8 \mathrm{~h}$ of recovery before neutropenia is induced.

From a therapeutic viewpoint, this study shows that antineutrophil strategies should be used before HI, or during the first 
Table 1. Rat brain energy metabolites after HI

\begin{tabular}{|c|c|c|c|c|c|c|}
\hline & $\mathrm{P}-\mathrm{Cr}$ & ATP & ADP & AMP & TAN & $\mathrm{EC}$ \\
\hline Normal $(n=10)$ & $2.64 \pm 0.32$ & $2.29 \pm 0.18$ & $0.39 \pm 0.13$ & $0.037 \pm 0.029$ & $2.72 \pm 0.22$ & $0.91 \pm 0.02$ \\
\hline \multicolumn{7}{|l|}{ Neutropenic } \\
\hline \multicolumn{7}{|l|}{ Ipsilateral } \\
\hline $0(n=16)$ & $1.18 \pm 0.70$ & $1.34 \pm 0.76$ & $0.44 \pm 0.15$ & $0.234 \pm 0.190$ & $2.02 \pm 0.56$ & $0.74 \pm 0.16$ \\
\hline $6(n=12)$ & $2.76 \pm 0.31$ & $2.29 \pm 0.22$ & $0.45 \pm 0.25$ & $0.043 \pm 0.028$ & $2.79 \pm 0.33$ & $0.91 \pm 0.04$ \\
\hline \multicolumn{7}{|l|}{ Contralateral } \\
\hline $6(n=12)$ & $2.96 \pm 0.41$ & $2.42 \pm 0.23$ & $0.51 \pm 0.20$ & $0.034 \pm 0.026$ & $2.96 \pm 0.29$ & $0.90 \pm 0.03$ \\
\hline \multicolumn{7}{|l|}{ Nonneutropenic } \\
\hline \multicolumn{7}{|l|}{ Ipsilateral } \\
\hline \multicolumn{7}{|l|}{ Recovery (h) } \\
\hline $0(n=16)$ & $0.96 \pm 0.57$ & $0.88 \pm 0.59$ & $0.41 \pm 0.14$ & $0.263 \pm 0.126$ & $1.55 \pm 0.56^{*}$ & $0.67 \pm 0.11$ \\
\hline $6(n=10)$ & $2.59 \pm 0.40$ & $2.12 \pm 0.27$ & $0.42 \pm 0.17$ & $0.050 \pm 0.032$ & $2.59 \pm 0.32$ & $0.90 \pm 0.03$ \\
\hline
\end{tabular}

Brain energy metabolites are shown for a group of normal 7-d-old rat pups and for a neutropenic and a non-neutropenic group of rats at 0 and $6 \mathrm{~h}$ after a $\mathrm{HI}$ insult to the right (ipsilateral) hemisphere. Values, expressed as mmol $/ \mathrm{kg}$, represent means $\pm \mathrm{SD}$ of cerebral hemispheres either ipsilateral or contralateral to the common carotid artery occlusion. P-Cr, phospho-creatinine; TAN, total adenine nucleotides (ATP + ADP + AMP); EC, energy charge [ATP + (0.5 * ADP)]/TAN.

$* p<0.05$, neutropenia $v s$ non-neutropenia TAN at $0 \mathrm{~h}$ recovery, Mann - Whitney $U$ test.

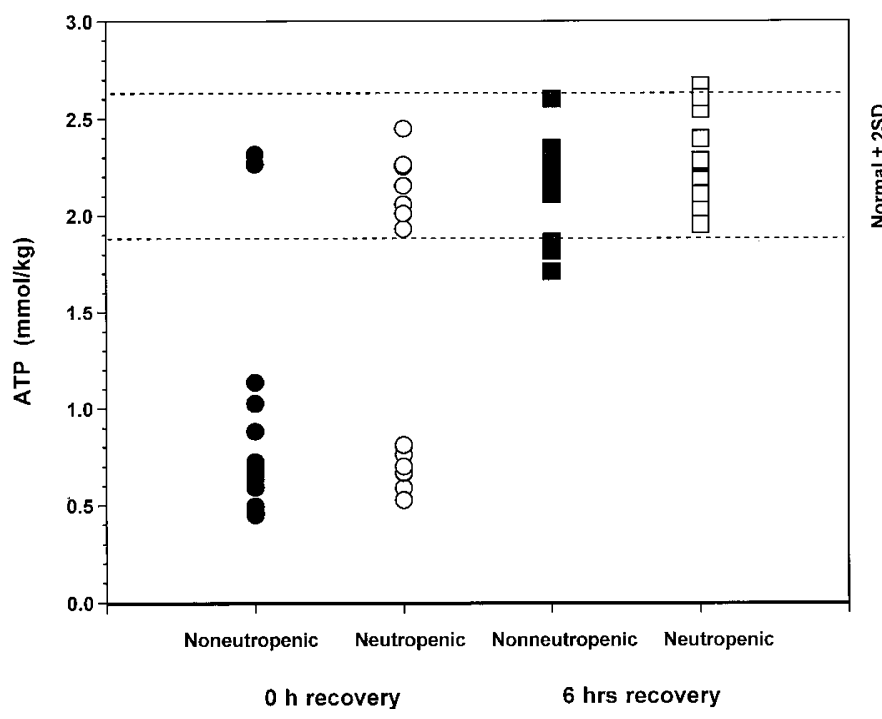

Figure 4. ATP levels measured in the ipsilateral hemisphere of neutropenic (solid symbols) and non-neutropenic (open symbols) rats at $0 \mathrm{~h}$ recovery from $\mathrm{HI}$ (circles) and at $6 \mathrm{~h}$ of recovery (squares). The normal range is defined by the dotted lines. At $0 \mathrm{~h}$ recovery, more neutropenic rats had ATP levels in the normal range $\left(p=0.04, \chi^{2}\right)$ vs non-neutropenic $(p=0.06$, Mann-Whitney $U$ test). ATP levels were largely within the normal range at $6 \mathrm{~h}$ recovery in both groups.

few hours of recovery. We found that virtually eliminating circulating neutrophils by $4-8 \mathrm{~h}$ of recovery was not protective, so it is unlikely that infiltrating neutrophils contribute to injury in the neonatal rat. It is also unlikely that any antineutrophil strategy will be successful after the first $6 \mathrm{~h}$ of recovery in the neonate. This differs from stroke studies in adult rodent models where antineutrophil strategies were initiated hours after recovery and where it is still felt that neutrophils are important contributors to secondary vascular and parenchymal injury after cerebral ischemia.

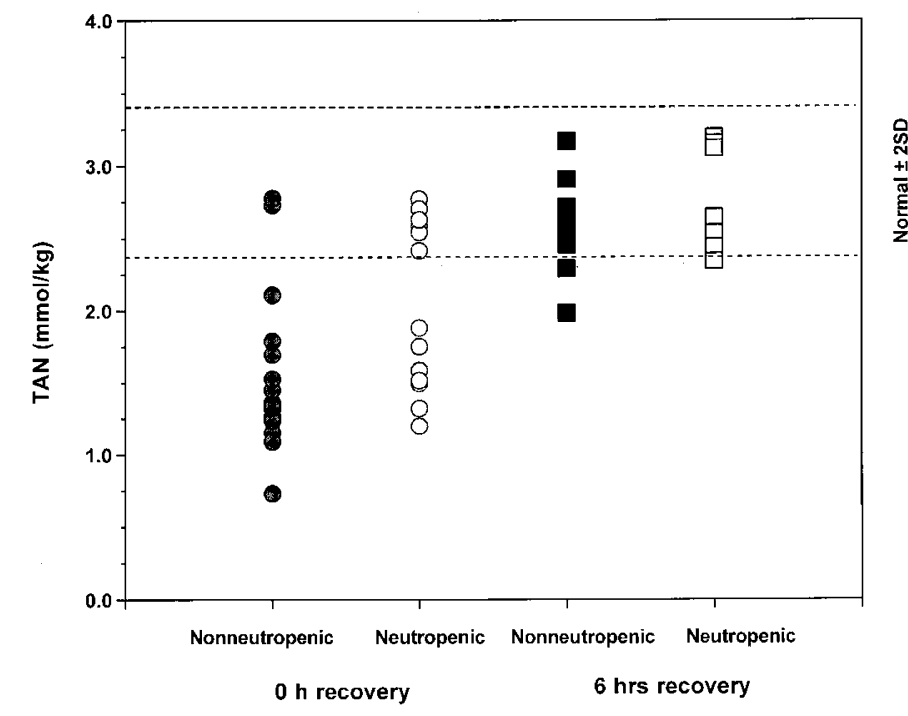

Figure 5. TAN in ipsilateral hemisphere of neutropenic (solid symbols) and non-neutropenic (open symbols) rats at $0 \mathrm{~h}$ recovery from $\mathrm{HI}$ (circles) and at $6 \mathrm{~h}$ of recovery (squares). The normal range is defined by the dotted lines. At $0 \mathrm{~h}$ recovery, more neutropenic rats had higher TAN levels $v s$ non-neutropenic rats $(p=0.03$, Mann-Whitney $U$ test). TAN levels were largely within normal at $6 \mathrm{~h}$ recovery in both groups.

Table 2. Effect of Antineutrophil serum on rectal temperature during hypoxia

\begin{tabular}{lccc}
\hline & \multicolumn{3}{c}{ Measurement periods } \\
\cline { 2 - 4 } Treatment group & Before hypoxia & During hypoxia & After hypoxia \\
\hline Neutropenic & $36.9 \pm 1.1$ & $36.8 \pm 1.2$ & $37.1 \pm 1.1$ \\
Control & $36.9 \pm 0.7$ & $36.4 \pm 0.7$ & $37.1 \pm 0.9$ \\
\hline
\end{tabular}

Temperature measurments taken before, during, and after hypoxia in six rats rendered neutropenic with ANS compared with six normal rats. For each rat, we calculated the average temperature derived from three to six measurements taken during the 30-60-min measurement period.

Results are mean $\pm \mathrm{SD}$. No temperature differences were seen. 
Knowing that neutrophils must produce their damaging effects either during the insult or in the first few hours of recovery indicates that they achieve these effects from the intravascular compartment, as they have not had time to extravasate. Even in adult animal models neutrophils are unlikely to extravasate before the first $6 \mathrm{~h}$ of recovery. In neonatal animals, neutrophil extravasation is even less likely during this interval. We and others were unable to show that neutrophils accumulate in the damaged brain to the extent seen in adults, and certainly not in the first $6 \mathrm{~h}(19,20)$. Parenchymal accumulation has been reported by some investigators to begin from $12-24 \mathrm{~h}(20,21)$. We have not been able to detect neutrophil extravasation in noninfarcted tissue using either stains for myeloperoxidase or using antibodies that detect neutrophils $(19,32)$.

From the intravascular compartment neutrophils can reduce the flow of red blood cells during low flow states $(23,33,34)$. In the immature rat pup model we used, blood flow to the hemisphere ipsilateral to carotid ligation is reduced while it is maintained in most of the contralateral hemisphere (35). Morphologic damage is largely confined to the right (ipsilateral) hemisphere allowing the contralateral hemisphere to act as a reference for morphometric analysis or atrophy (29). Hence, ischemia is necessary to produce damage, and hypoxia alone is not damaging in this model. Accordingly, factors that aggravate local ischemia will enhance the primary insult.

Neutrophils are relatively large, stiff cells that are attracted to roll along the vascular endothelium by the rapid expression of selectins on endothelial cells and on the neutrophil. Hypoxia alone increases adherence to endothelial cells (36). Neutrophil rolling is followed by expression of adhesion molecules that fix the neutrophil to the vessel wall $(2,23,34)$. Neutrophils can generate oxygen free radicals and release lysosomal enzymes, myeloperoxidase, proteases, and other mediators that promote vascular injury and thrombosis. Superoxide produced by neutrophils and endothelial cells during reperfusion promotes adherence, but it is inactivated by binding to nitric oxide (37). During cerebral ischemia there is an inhibition of nitric oxide production which enhances neutrophil-endothelial adherence (38).

Neutrophil-endothelial cell interaction mediated by adhesion molecules has also been shown to induce vasoconstriction (39, 40). Thus, neutrophils may mediate tissue damage in the brain by impairing microvascular function and they may cause damage by the cytotoxic mediators that they release. In some ischemic tissue, granulocyte plugging is not relieved by reperfusion and a reduction in flow persists, producing what has been called the "no-reflow" phenomenon in the microcirculation (2). In the immature rat pup model we used in this study, blood flow returns to normal after hypoxia, and delayed hypoperfusion has not been reported using radioisotope tracers (35, 41).

We postulated that neutrophils might impair red cell flow and oxygen transport during ischemia and/or during early recovery. Accordingly, we evaluated energy metabolism in both hemispheres at the end of hypoxia ( $0 \mathrm{~h}$ recovery) and $6 \mathrm{~h}$ later. We found that TAN and, to some extent, ATP were better preserved in the neutropenic hemispheres at the end of hypoxia
(Fig. 5, Table 1). These data support the notion that neutrophils impaired red cell movement and oxygen transfer. Interestingly, energy metabolites were restored to normal in neutropenic and non-neutropenic rats at $6 \mathrm{~h}$ of recovery. This suggests that red cell trafficking had recovered by $6 \mathrm{~h}$. We have shown previously that energy metabolites may still be impaired at $4 \mathrm{~h}$ of recovery (25). The restoration of energy metabolism seen at $6 \mathrm{~h}$ is in keeping with other studies evaluating energy metabolism in the newborn rat (42). Energy metabolites return to baseline, then 1-2 d later there is another fall in high-energy phosphates, sometimes referred to as secondary energy failure, the extent of which correlates with cerebral infarction $(25,42)$.

The severity of the brain injury in this model is related to the severity of the energy failure during the primary insult (43). It is probable that the preservation in energy metabolism in the neutropenic rats at the end of hypoxia was sufficient to account for the difference between the early (brain swelling) and late indicators (atrophy) of injury. Vasthare et al. (44) showed that leukopenia resulted in preservation of brain electrical activity during rat brain ischemia.

In this study, we assessed cerebral blood flow in a group of eight neutropenic 7-d-old rats with the radioisotope technique using iodoantipyrine (35). We found no difference in the reduction of flow seen in the ipsilateral hemisphere at the end of $2.25 \mathrm{~h}$ ischemia, compared with a group of seven rats with normal neutrophil counts (data not shown). This finding may still be compatible with an impairment of red cell trafficking, as the radioisotope is completely distributed in the water compartment and plasma. Studies using intravital dye microscopic techniques have shown that, once there is a neutrophil plug in a capillary only plasma passes around the neutrophil, and red blood cells stack behind the neutrophil plug (45).

This study has important clinical implications for sick newborn infants who are subjected to conditions that can increase inflammatory cytokines in the cerebral vasculature, including sepsis, feto-maternal infection (46), and ischemia reperfusion injury (20). Children with cerebral palsy were shown to have elevated inflammatory cytokines and coagulation factors present in their cord blood samples (47). Circulating inflammatory cytokines will activate neutrophils and endothelium [reviewed in (48)]. In premature babies, chorioamnionitis can be associated with placental villous edema that may impair placental gas exchange (49). Activated neutrophils can impair cerebral blood flow even in the absence of ischemia (50).

Epidemiologic data indicates that feto-maternal infection, accompanied by vasculitis or thrombosis of fetal umbilical vessels, is associated with white matter brain injury in premature infants (51-53). Experimentally induced intrauterine infection causes brain damage in neonatal rabbits $(54,55)$, and the administration of endotoxin to neonatal animals is associated with injury to the periventricular white matter $(56,57)$. Notably, the periventricular white matter has very low blood flow even under normal conditions and the density of blood vessels in the white matter is lowest at around 28 wk gestation, about the same time that incidence of periventricular leukomalacia peaks $(58,59)$.

The presence of neutrophils in the umbilical vessels has a particularly strong correlation with neonatal sepsis and mor- 
bidity (52). Infants born prematurely with histologic chorioamnionitis have higher peripheral neutrophil counts (60), and intrauterine infection is associated with an elevation of inflammatory cytokines (46) that activate neutrophils and the vascular endothelium, thereby facilitating neutrophil "rolling" and adhesion. Mice deficient in key adhesion molecules are protected from ischemic injury $(61,62)$. Dammann and Leviton (63) have proposed that proinflammatory cytokines might be the link between prenatal intrauterine infection and neonatal brain damage, although there is also strong support of an ischemic component to the pathogenesis of PVL $(58,64)$. We speculate that activated neutrophil-endothelial interactions could be enhanced by both components, linking inflammatory cytokines with hypoperfusion mechanistically in a common pathway toward "vascular failure" in the pathogenesis of PVL. Paul et al. (65) observed that premature infants who develop an intraventricular hemorrhage have higher neutrophil counts in the first $3 \mathrm{~d}$ of life. Neutrophil-induced microvascular dysfunction will be even more likely if hypotension and cerebral hypoperfusion occur in the very susceptible sick premature infant. Accordingly, brain injury might be reduced by approaches that maintain cerebral perfusion (66), that reduce the effect of circulating inflammatory cytokines, and that reduce neutrophil-endothelial activation/adhesion (67).

In conclusion, this study demonstrates that neutrophils contribute to long-term hypoxic-ischemic brain injury in the 7-dold rat. It limits the timing of neutrophil involvement to the period of ischemia or the first few hours of recovery. It also reveals that neutrophils produce their damaging effects from within blood vessels, suggesting that they induce microvascular dysfunction, probably by impairing red cell flow, oxygen transport, and energy metabolism during ischemia. The clinical implications of these findings are particularly relevant to sick premature infants with elevated inflammatory cytokines in circulation, suggesting that activated neutrophil-endothelial interactions may warrant attention as a target for therapeutic intervention.

Acknowledgment. The authors thank Robert Brucklacher and Robert C. Vannucci for their assistance with the blood flow measurements.

\section{REFERENCES}

1. del Zoppo GJ, Garcia J 1995 Polymorphonuclear leukocyte adhesion in cerebrovascular ischemia. In: Granger DN, Schmid-Shonbein GW (eds) Physiology and Pathophysiology of Leukocyte Adhesion. Oxford University Press, New York, pp 408-433

2. Schmid-Schonbein G 1987 Capillary plugging by granulocytes and the no-flow phenomenon in the microcirculation. Fed Proc 46:2397-2401

3. Kochanek PM, Hallenbeck JM 1992 Polymorphonuclear leukocytes and monocytes/ macrophages in the pathogenesis of cerebral ischemia and stroke. Stroke 23:13671379

4. Matsuo Y, Kihara T, Ikeda M, Ninomiya M, Onodera H, Kogure K 1995 Role of neutrophils in radical production during ischemia and reperfusion of the rat brain: effect of neutrophil depletion on extracellular ascorbyl radical formation. J Cereb Blood Flow Metab 15:941-947

5. Garcia J, Liu K, Yoshida Y, Lian J, Chen S, del Zoppo G 1994 Influx of leukocytes and platelets in an evolving brain infarct (Wistar rat). Am J Pathol 144:188-199

6. Hallenbeck JM, Dutka AJ, Tanishima T, Kochanek PM, Kumaroo KK, Thompson CB, Obrenovitch TP, Contreras TJ 1986 Polymorphonuclear leukocyte accumulation in brain regions with low blood flow during the early postischemic period. Stroke $17: 246-253$

7. Zhang RL, Chopp M, Chen H, Garcia JH 1994 Temporal profile of ischemic tissue damage, neutrophil response, and vascular plugging following permanent and transient (2h) middle cerebral artery occlusion in the rat. J Neurol Sci 125:3-10
8. Dereski MO, Chopp M, Knight RA, Chen H, Garcia JH 1992 Focal cerebral ischemia in the rat: temporal profile of neutrophil responses. Neurosci Res Commmun 11:179186

9. Matsuo Y, Onodera H, Shiga Y, Nakamura M, Ninomiya M, Kihara T, Kogure K 1994 Correlation between myeloperoxidase-quantified neutrophil accumulation and ischemic brain injury in the rat: effects of neutrophil depletion. Stroke 25:1469-1475

10. Jiang N, Chopp M, Chahwala S 1998 Neutrophil inhibitory factor treatment of focal cerebral ischemia in the rat. Brain Res 788:25-34

11. Jiang N, Moyle M, Soule HR, Rote WE, Chopp M 1995 Neutrophil inhibitory factor is neuroprotective after focal ischemia in rats. Ann Neurol 38:935-942

12. Zhang RL, Chopp M, Jiang N, Tang WX, Prostak J, Manning AM, Anderson DC 1995 Anti-intercellular adhesion molecule-1 antibody reduces ischemic cell damage after transient but not permanent middle cerebral artery occlusion in the Wistar rat. Stroke 26:1438-1442

13. Anderson DC, Hughes BJ, Smith CW 1981 Abnormal mobility of neonatal polymorphonuclear leukocytes. J Clin Invest 68:863-874

14. Anderson DC, Rothlein R, Marlin SD, Krater SS, Smith CW 1990 Impaired transendothelial migration by neonatal neutrophils: abnormalities of mac-1 (cd11b/cd18)dependent adherence reactions. Blood 76:2613-2621

15. Krause PJ, Herson VC, Boutin-Lebowitz J, Eisenfeld L, Block C, LoBello T, Maderazo EG 1986 Polymorphonuclear leukocyte adherence and chemotaxis in stressed and healthy neonates. Pediatr Res 20:296-300

16. Torok C, Lundahl J, Hed J, Lagercrantz H 1993 Diversity in regulation of adhesion molecules (mac-1 and 1-selectin) in monocytes and neutrophils from neonates and adults. Arch Dis Child 68:561-565

17. Anderson DC, Hughes BJ, Smith CW 1981 Abnormal mobility of neonatal polymorphonuclear leukocytes. Relationship to impaired redistribution of surface adhesion sites by chemotactic factor or colchicine. J Clin Invest 68:863-874

18. Jones DH, Schmalstieg FC, Dempsey K, Krater SS, Nannen DD, Smith CW, Anderson DC 1990 Subcellular distribution and mobilization of mac-1 (cd11b/cd18) in neonatal neutrophils. Blood 75:488-498

19. Hudome S, Palmer C, Roberts RL, Mauger D, Housman C, Towfighi J 1997 The role of neutrophils in the production of hypoxic-ischemic brain injury in the neonatal rat. Pediatr Res 41:607-616

20. Bona E, Andersson A, Blomgren K, Gilland E, Puka-Sundvall M, Gustafson K, Hagberg H 1999 Chemokine and inflammatory cell response to hypoxia-ischemia in immature rats. Pediatr Res 45:500-509

21. Benjelloun N, Renolleau S, Represa A, Ben-Ari Y, Charriaut-Marlangue C 1999 Inflammatory responses in the cerebral cortex after ischemia in the p7 neonatal rat. Stroke 30:1916-1923

22. Viswanath M, Palmer C, Roberts RL 1997 Reduction of hypoxic ischemic brain swelling in the neonatal rat with paf antagonist web2170: lack of long term protection. Pediatr Res 48:109-113

23. Schmid-Schonbein GW, Lee J 1995 Leukocytes in capillary flow. Int J Microcirc Clin Exp 15:255-264

24. Vannucci RC, Lyons DL, Vasta F 1988 Regional cerebral blood flow during hypoxiaischemia in immature rats. Stroke 19:245-250

25. Palmer C, Brucklacher RM, Christensen MA, Vannucci RC 1990 Carbohydrate and energy metabolism during the evolution of hypoxic-ischemic brain damage in the immature rat. J Cereb Blood Flow Metab 10:227-235

26. Northington FJ, Ferriero DM, Graham EM, Traystman RJ, Martin LJ 2001 Early neurodegeneration after hypoxia-ischemia in neonatal rat is necrosis while delayed neuronal death is apoptosis. Neurobiol Dis 8:207-219

27. Rice JE, Vannucci RC, Brierley JB 1981 The influence of immaturity on hypoxicischemic brain damage in the rat. Ann Neurol 9:131-141

28. Palmer C, Towfighi J, Roberts RL, Heitjan DF 1993 Allopurinol administered after inducing hypoxia-ischemia reduces brain injury in 7-day-old rats. Pediatr Res 33:405-411

29. Towfighi J, Housman C, Vannucci RC, Heitjan DF 1994 Effect of unilateral perinatal hypoxic-ischemic brain damage on the gross development of opposite cerebral hemisphere. Biol Neonate 65:108-118

30. Vannucci RC, Duffy TE 1974 Influence of birth on carbohydrate and energy metabolism in rat brain. Am J Physiol 226:933-940

31. Lowry O, Passonneau J, Hasselberger F, Schulz D 1964 Effect of ischemia on known substrates and cofactors of the glycolytic pathway in brain. J Biol Chem 239:18-30

32. Towfighi J, Yager JY, Housman C, Vannucci RC 1991 Neuropathology of remote hypoxic-ischemic damage in the immature rat. Acta Neuropathol 81:578-587

33. Schmid-Schonbein GW, Usami S, Skalak R, Chien S 1980 The interaction of leukocytes and erythrocytes in capillary and postcapillary vessels. Microvasc Res 19:45-70

34. Del Zoppo GJ, Schmid-Schonbein GW, Mori E, Copeland BR, Chang CM 1991 Polymorphonuclear leukocytes occlude capillaries following middle cerebral artery occlusion and reperfusion in baboons. Stroke 22:1276-1283

35. Ringel M, Bryan RM, Vannucci RC 1991 Regional cerebral blood flow during hypoxia-ischemia in the immature rat: comparison of iodoantipyrine and iodoamphetamine as radioactive tracers. Brain Res Dev Brain Res 59:231-235

36. Ginis I, Mentzer SJ, Li X, Faller DV 1995 Characterization of a hypoxia-responsive adhesion molecule for leukocytes on human endothelial cells. J Immunol 155:802-810

37. Beckman JS 1998 Interactions of oxidants, nitric oxide, and antioxidant defenses in cerebral ischemia and injury. In: Ginsberg MD, Bogousslavsky J (eds) Cerebrovascular Disease: Pathophysiology, Diagnosis, and Management. Blackwell Science, Malden, MA, pp 455-470

38. Gidday JM, Park TS, Shah AR, Gonzales ER 1998 Modulation of basal and postischemic leukocyte-endothelial adherence by nitric oxide. Stroke 29:1423-1430 
39. Murohara T, Buerke M, Lefer AM 1994 Polymorphonuclear leukocyte-induced vasocontraction and endothelial dysfunction: role of selectins. Arterioscler Thromb 14:1509-1519

40. Minamino T, Kitakaze M, Node K, Funaya H, Inoue M, Hori M, Kamada T 1996 Activated polymorphonuclear leukocytes induce constriction of canine coronary artery via mac-1, but not lfa-1, and icam-1. J Mol Cell Cardiol 28:1575-1581

41. Mujsce DJ, Christensen MA, Vannucci RC 1990 Cerebral blood flow and edema in perinatal hypoxic-ischemic brain damage. Pediatr Res 27:450-453

42. Blumberg RM, Cady EB, Wigglesworth JS, McKenzie JE, Edwards AD 1997 Relation between delayed impairment of cerebral energy metabolism and infarction following transient focal hypoxia-ischaemia in the developing brain. Exp Brain Res 113:130-137

43. Williams GD, Towfighi J, Smith MB 1994 Cerebral energy metabolism during hypoxia-ischemia correlates with brain damage: A 31p NMR study in unanesthetized immature rats. Neurosci Lett 170:31-34

44. Vasthare U, Heinel L, Rosenwasser R, Tuma R 1990 Leukocyte involvement in cerebral ischemia and reperfusion injury. Surg Neurol 33:261-265

45. Bienvenu K, Granger DN 1993 Leukocyte adhesion in ischemia/reperfusion. Blood Cells 19:279-289

46. Gomez R, Romero R, Ghezzi F, Yoon BH, Mazor M, Berry SM 1998 The fetal inflammatory response syndrome. Am J Obstet Gynecol 179:194-202

47. Nelson KB, Dambrosia JM, Grether JK, Phillips TM 1998 Neonatal cytokines and coagulation factors in children with cerebral palsy. Ann Neurol 44:665-675

48. Palmer C 1995 Hypoxic-ischemic encephalopathy. Therapeutic approaches against microvascular injury, and role of neutrophils, PAF, and free radicals. Clin Perinatol 22:481-517

49. Naeye RL, Maisels MJ, Lorenz RP, Botti JJ 1983 The clinical significance of placental villous edema. Pediatrics 71:588-594

50. Chen L, Vicaut E, Sercombe R 2002 Polymorphonuclear leukocyte activation induces cerebral hypoperfusion in rats in the absence of previous ischemia-reperfusion damage. Neurosci Lett 331:203-207

51. Leviton A, Paneth N, Reuss ML, Susser M, Allred EN, Dammann O, Kuban K, Van Marter LJ, Pagano M 1999 Maternal infection, fetal inflammatory response, and brain damage in very low birth weight infants. Pediatr Res 46:566-575

52. Baud O, Emilie D, Pelletier E, Lacaze-Masmonteil T, Zupan V, Fernandez H, Dehan M, Frydman R, Ville Y 1999 Amniotic fluid concentrations of interleukin-1 beta, interleukin-6 and TNF-alpha in chorioamnionitis before 32 weeks of gestation: histological associations and neonatal outcome. Br J Obstet Gynaeco 106:72-77
53. Redline RW, Wilson-Costello D, Borawski E, Fanaroff AA, Hack M 1998 Placental lesions associated with neurologic impairment and cerebral palsy in very low-birthweight infants. Arch Pathol Lab Med 122:1091-1098

54. Yoon BH, Romero R, Jun JKJ, Park KH, Park JD, Ghezzi F, Kim BI 1997 Amniotic fluid cytokines (interleukin-6, tumor necrosis factor- $\alpha$, interleukin- $1 \beta$, and interleukin-8) and the risk for the development of bronchopulmonary dysplasia. Am J Obstet Gynecol 177:825-830

55. Yoon BH, Kim CJ, Romero R, Jun JK, Park KH, Choi ST, Chi JG 1997 Experimentally induced intrauterine infection causes fetal brain white matter lesions in rabbits. Am J Obstet Gynecol 177:797-802

56. Gilles FH, Averill DR, Kerr CS 1977 Neonatal endotoxin encephalopathy. Ann Neurol 2:49-56

57. Young RSK, Yagel SK, Towfighi J 1983 Systemic and neuropathologic effects of $E$. coli endotoxin in neonatal dogs. Pediatr Res 17:349-353

58. Volpe JJ 2001 Neurobiology of periventricular leukomalacia in the premature infant Pediatr Res 50:553-562

59. Miyawaki T, Matsui K, Takashima S 1998 Developmental characteristics of vessel density in the human fetal and infant brains. Early Hum Dev 53:65-72

60. Watterberg KL, Scott SM, Naeye RL 1997 Chorioamnionitis, cortisol, and acute lung disease in very low birth weight infants. Pediatrics 99:E6

61. Connolly Jr ES, Winfree CJ, Springer TA, Naka Y, Liao H, Yan SD, Stern DM, Solomon RA, Gutierrez-Ramos JC, Pinsky DJ 1996 Cerebral protection in homozygous null ICAM-1 mice after middle cerebral artery occlusion - role of neutrophil adhesion in the pathogenesis of stroke. J Clin Invest 97:209-216

62. Prestigiacomo CJ, Kim SC, Connolly ES, Liao H, Yan SF, Pinsky DJ 1999 CD18 mediated neutrophil recruitment contributes to the pathogenesis of reperfused but not nonreperfused stroke. Stroke 30:1110-1116

63. Dammann O, Leviton A 1997 Maternal intrauterine infection, cytokines, and brain damage in the preterm newborn. Pediatr Res 42:1-8

64. Dammann O, Allred EN, Kuban KCK, Van Marter LJ, Stewart JE, Pagano M, Leviton A 2001 Hypocarbia during the first 24 postnatal hours and white matter echolucencies in newborns $<$ or $=28$ weeks gestation. Pediatr Res 49:388-393

65. Paul DA, Leef KH, Stefano JL 2000 Increased leukocytes in infants with intraventricular hemorrhage. Pediatr Neurol 22:194-199

66. Volpe JJ 1997 Brain injury in the premature infant-from pathogenesis to prevention. Brain Dev 19:519-534

67. Eun BL, Liu XH, Barks JDE 2000 Pentoxifylline attenuates hypoxic-ischemic brain injury in immature rats. Pediatr Res 47:73-78 